\title{
A New Approach for Real-Time Voltage Stability Monitoring Using PMUs
}

\author{
Kai Sun, Fengkai Hu, \\ University of Tennessee \\ Knoxville, TN, USA \\ kaisun@utk.edu, fengkaihu@utk.edu
}

\author{
Navin Bhatt \\ Electric Power Research Institute, USA \\ nbhatt@epri.com
}

\begin{abstract}
This paper proposes a new approach using phasor measurement units (PMUs) to estimate real-time voltage stability margin for a load area supported by multiple tie lines. For the monitored tie lines, it can provide accurate real and reactive power transfer margins. Compared to a traditional Thevenin equivalent-based approach, the new approach is more tolerant of fluctuations in the phase angles of the external system and the power factors of tie-line flows. A two-bus power system and the IEEE 39-bus system are used to test this new approach and compare it with the traditional approach.
\end{abstract}

Index Terms-Phasor measurement unit; voltage security assessment; voltage stability; voltage collapse

\section{INTRODUCTION}

$\mathrm{V}$ OLTAGE stability is a major concern in power system operations and a leading factor to limit power transfers. Voltage instability usually starts from a local bus but may also happen on boundary buses of a load center area that receive power from the external system through heavily loaded tie lines. Traditionally, steady-state and transient voltage security issues can respectively be identified by voltage security assessment (VSA) tools using power-flow analysis and time-domain simulation. If integrated into the energy management system (EMS), those tools can tell which contingencies may cause voltage insecurity under current operating condition. However, the accuracies of those VSA tools are limited by several factors: 1) they rely on accurate power system models; 2) they require a convergent state estimate on the current operating condition, which, however, may be unavailable under stressed conditions; 3) the contingencies to be analyzed are limited and can never cover all possible disturbances.

To complement those model-based VSA tools, some literatures have proposed measurement-based VSA methods to only utilize measurement data to either estimate real-time voltage stability margin [1]-[10] or predict voltage security following anticipant contingencies [11]. Most of the methods for estimating real-time voltage stability margin are for a load-pocket area fed by remote generation through a

This material is based upon work supported by the Electric Power Research Institute and the CURENT Engineering Research Center transmission corridor. Thus, a typical approach is to regard the load pocket area as a load bus and the external system the remote generation directly supporting the load area) as a Thevenin equivalent having a constant voltage source (denoted by $\bar{E}=E \angle \theta$ ) connected through a constant impedance (denoted by $\bar{Z}_{T}=Z_{T} \angle \beta$ ) as shown by Fig. 1 . With real-time voltage and current measurements on the sending or receiving end of the transmission corridor over a time window, the equivalent load impedance (denoted by $\bar{Z}_{L}$ ) and the Thevein parameters (i.e. $Z_{T}, \beta, E$ and $\theta$ ) can be estimated. Then, the power transfer limit in terms of voltage stability for that transmission corridor is met if

$$
\left|\bar{Z}_{L}\right|=\left|\bar{Z}_{T}\right|
$$

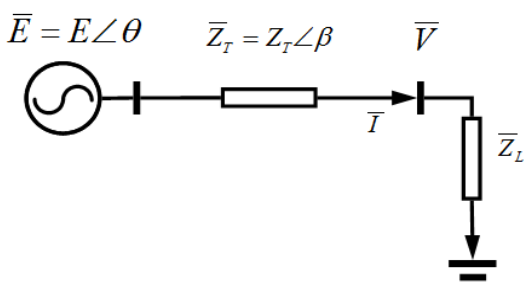

Fig. 1. Thevenin Equivalent

Fig. 2. Non-pocket load area

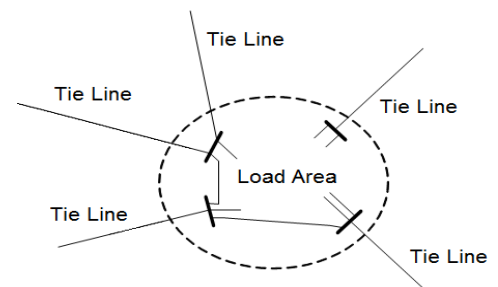

In practice, that approach may not be accurate in monitoring voltage stability for a non-pocket load area fed by multiple tie lines from different directions as illustrated by Fig. 2. One reason is that the assumption of the Thevenin voltage phasor (i.e. $E$ and $\theta$ ) being constant requires real-time 
synchronized phasor measurements on both the load area and the external system. When the approach is applied to a radial system or a load-pocket area, those synchronized phasor measurements can easily be obtained by placing phasor measurement units (PMUs) on both ends of the transmission corridor and then using the phase angle on the generation side as a reference to calculate relative angles of the phasor measurements on the load side, i.e. $\theta=$ constant. However, when the approach is applied to a non-pocket load area, there are usually multiple tie lines from different generation areas, so it is not as easy to directly measure the angle difference between the generation and the load sides. PMUs can still be placed on boundary buses of the load area to measure the angles of the load sides relative to a specific common reference of the entire system, which is, however, unnecessarily near any of the generation areas supporting the load area. Thus, a new approach is needed to relax the “constant $\theta$ ' assumption on the Thevenin voltage source. We may still assume its constant magnitude $E$ over a time window if the external system has very stable voltage profile.

Another problem with the traditional Thevenin equivalentbased approach is based on the equality (1) to predict voltage instability. Actually, the real and reactive powers transferred to the load area may meet their limits at different times if the power factors of the tie line flows are not constant. It is expected to assume fluctuating power factors and define voltage stability indices separately for real and reactive power margins or even for individual tie lines.

This paper studies voltage stability monitoring for a nonpocket area using real-time PMU measurements on its boundary buses and proposes a new approach to meet the aforementioned requirements. The approach is tested by case studies on a two-bus system with a floating angle of the voltage source and then the IEEE 39-bus power system.

\section{Proposed Approach}

For a load area as illustrated by Fig. 2, PMUs are assumed to be placed on its boundary buses to measure real-time voltage phasors there and the real-time current phasors of the tie lines connected (in fact, the case study presented later on the IEEE 39-bus system indicates that the new approach does not need to monitor all boundary buses and tie lines in order to predict voltage instability as long as the most critical boundary buses and tie lines are monitored by PMUs). The boundary-bus voltage phasors $\bar{V}_{i}$ and tie-line complex powers $\bar{S}_{i}$ are available from real-time PMU measurements.

The monitored boundary buses can be represented by one fictitious bus with voltage phasor $\bar{V}$ calculated by:

$$
\bar{V}=\frac{\bar{S}}{\bar{I}^{*}}=\bar{S} / \sum_{i} \frac{\overline{S_{i}}}{\overline{V_{i}}} \quad \text { where } \bar{S}=\sum_{i} \overline{S_{i}}
$$

$\bar{I}$ denotes the total current of the monitored tie lines. Define
$\bar{Z}_{L}=Z_{L} \angle \varphi=\bar{V} / \bar{I}$. The proposed approach aims at utilizing measurements of $\bar{V}$ and $\bar{I}$ (or equivalently, $\bar{S}$ ) over a time window to estimate Thevenin equivalent parameters $Z_{T}, \beta$ and $E$ in Fig. 1, which are assumed to be constant over that time window. Here, $\theta$ is relaxed as discussed above.

The proposed approach conducts the following steps:

i) Obtain synchronized voltage and current phasor measurements from PMUs at the monitored boundary buses, and calculate $\bar{V}, \bar{I}$ and then $\bar{Z}_{L}$ in real time

ii) Estimate $Z_{T}, \beta$ and $E$ using the measurements over the latest time window of $T$

iii) Calculate the security indices defined later, e.g. the transfer limits (denoted by $S_{\max }, P_{\max }$ and $Q_{\max }$ ) of the total tie-line apparent, real and reactive power flows and the voltage security limits corresponding to those transfer limits (denoted by $V_{\min }^{S}, V^{P}{ }_{\min }$, and $V^{Q}{ }_{\min }$ ).

Note that $S_{\max }, P_{\max }$ and $Q_{\max }$ (or $V_{\min }^{S}, V^{P}$ min , and $\left.V^{Q}{ }_{\min }\right)$ may be met at different times as illustrated by Fig. 3, indicating that one could be a more limiting factor on voltage stability than the others.

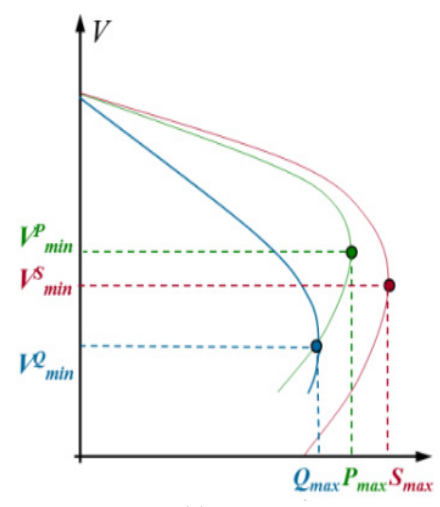

(a)

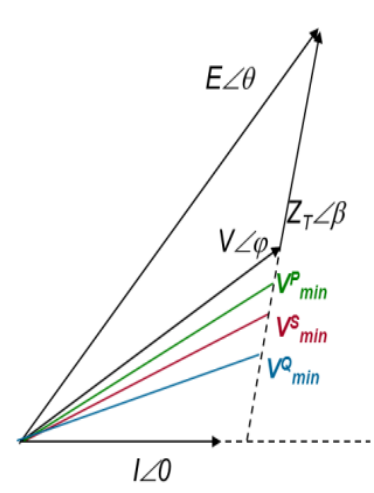

(b)
Fig. 3. Security indices

Thevenin parameters are estimated by the following procedure over a time window with $N$ measuring points at $t_{1} \sim t_{N}$. As shown in Fig. 3(b), if the current phasor $\bar{I}$ is the reference, there are:

$$
E \angle \theta\left(t_{i}\right)=I\left(t_{i}\right) \times Z_{T} \angle \beta+V\left(t_{i}\right) \angle \varphi\left(t_{i}\right), \quad i=1 \sim N
$$

Let $\alpha=\theta\left(t_{N}\right)-\theta\left(t_{1}\right)$ and define

$$
\begin{gathered}
\bar{Z}_{T}(\alpha)=Z_{T}(\alpha) \angle \beta(\alpha)=-\frac{\angle \alpha \times V\left(t_{N}\right) \angle \phi\left(t_{N}\right)-V\left(t_{1}\right) \angle \phi\left(t_{1}\right)}{\angle \alpha \times I\left(t_{N}\right)-I\left(t_{1}\right)} \\
\bar{E}_{i}(\alpha)=I\left(t_{1}\right) \times \bar{Z}_{T}(\alpha)+V\left(t_{i}\right) \angle \phi\left(t_{i}\right), \quad i=1 \sim N
\end{gathered}
$$

Find $\alpha$ to minimize $\operatorname{var}\left\{E_{i} \mid i=1 \sim N\right\}$. Then, $E, Z_{T}$ and $\beta$ over the time window can be solved by (4) and (5).

When the total apparent power $S$ of the monitored tie lines meets $S_{\max }, V \times I$ is constant despite changes of $V$ or $I$. Accordingly, $\ln V+\ln I$ is constant, so $d V / V+d I / I=0$, i.e. $V / I=-d V / d I$. Therefore, once $Z_{L}=-d V / d I, S$ meets $S_{\max }$. 
Similarly, $P=V \times I \times \cos \varphi$ or $Q=V \times I \times \sin \varphi$ is constant if $P$ or $Q$ meets $P_{\max }$ or $Q_{\max }$. Thus, define impedance indices $Z_{S}, Z_{P}$ and $Z_{Q}$ as follows to be security limits of $Z_{L}$ in terms of the power transfer limits of $S, P$ and $Q$.

$$
Z_{L}= \begin{cases}-\frac{d V}{d I} \stackrel{\text { def }}{=} Z_{S} & \text { when } S=S_{\max } \\ -\frac{d V}{d I}+V \tan \varphi \frac{d \varphi}{d I} \stackrel{\text { def }}{=} Z_{P} & \text { when } P=P_{\max } \\ -\frac{d V}{d I}-V \cot \varphi \frac{d \varphi}{d I} \stackrel{d e f}{=} Z_{Q} & \text { when } Q=Q_{\max }\end{cases}
$$

From (6), only when $\varphi$ is constant, there are

$$
\begin{aligned}
& Z_{S}=Z_{P}=Z_{Q} \\
& \bar{S}_{\text {max }} \stackrel{\Delta}{=} S_{\text {max }} \angle \phi=P_{\text {max }}+j Q_{\text {max }}
\end{aligned}
$$

Otherwise, the total $S, P$ and $Q$ of the monitored tie lines may meet their limits at different times. Because, for a real-world power system, any of $S, P$ and $Q$ approaching the limit may cause the system to risk voltage instability, it is more advisable to estimate $S_{\max }, P_{\max }$ and $Q_{\max }$ separately to identify the limiting factor on voltage stability. Define an impedance index

$$
Z_{T}^{S}=Z_{S}+\left(Z_{T}-Z_{S}\right)\left|\frac{1-\left(Z_{S} / Z_{L}\right)^{r}}{1+\left(Z_{S} / Z_{L}\right)^{r}}\right|
$$

It equals $Z_{S}$ when $Z_{L}=Z_{S}$ and approaches $Z_{T}$ when $Z_{L}>$ or $<<Z_{S}$. Replacing $Z_{S}$ by $Z_{P}$ or $Z_{Q}$ in (9), indices $Z_{T}^{P}$ or $Z_{T}^{Q}$ can be defined. Impedance indices $Z_{T}^{S}, Z_{T}^{P}$ and $Z_{T}^{Q}$ are actually the limits of $Z_{L}$ corresponding respectively to $S_{\max }, P_{\max }$ and $Q_{\max }$. Here, $r \geq 0$ is configurable to make those limits stay around $Z_{T}$. If $r=0, Z_{S}, Z_{P}$ and $Z_{Q}$ are the limits. In the case studies below, $r=0$ is assumed as an example. Using those impedance indices, the following indices on stability limits and risks can be calculated. The formulas are easily obtained from relationship of the phasors in Fig. 3(b).

$$
\begin{gathered}
\left.V_{\text {min }}^{X} \stackrel{\text { def }}{=} V\right|_{Z_{L}=Z_{T}^{X}}=\frac{E Z_{T}^{X}}{\left|Z_{T}^{X} \angle \varphi+\bar{Z}_{T}\right|}, \text { where "X" is } P, Q \text { or } S \\
\left.S_{\max } \stackrel{\text { def }}{=} S\right|_{Z_{L}=Z_{T}^{S}}=\left.\frac{\left(V_{\text {min }}^{S}\right)^{2}}{Z_{T}^{S}} \quad P_{\max } \stackrel{\text { def }}{=} P\right|_{Z_{L}=Z_{T}^{P}}=\operatorname{Re}\left[\frac{\left(V_{\min }^{P}\right)^{2}}{\left(Z_{T}^{P} \angle \varphi\right)^{*}}\right] \\
\left.Q_{\max } \stackrel{\text { def }}{=} Q\right|_{Z_{L}=Z_{T}^{Q}}=\operatorname{Im}\left[\frac{\left(V_{\min }^{Q}\right)^{2}}{\left(Z_{T}^{O} \angle \varphi\right)^{*}}\right]
\end{gathered}
$$

The real-time risks of the total apparent, real and reactive powers reaching their limits are calculated respectively by

$$
\begin{aligned}
& R_{S} \stackrel{\text { def }}{=} \min \left(Z_{T}^{S} / Z_{L}, 1\right) \quad R_{P} \stackrel{\text { def }}{=} \min \left(Z_{T}^{P} / Z_{L}, 1\right) \\
& R_{Q} \stackrel{\text { def }}{=} \min \left(Z_{T}^{Q} / Z_{L}, 1\right)
\end{aligned}
$$

\section{CASE STUdiES}

The proposed approach (referred as the "new approach") is compared to a traditional Thevenin equivalent based approach (referred as the "old approach") that assumes constant Thevenin parameters $E, \theta, Z_{T}$ and $\beta$ and solves those parameters by the least square method. The old approach calculates the indices in (10) and (11) by letting $Z_{L}=Z_{T}$ and an risk index equal to $Z_{T} / Z_{L}$ if $Z_{L}>Z_{T}$ or 1 , otherwise.

\section{A. Two-bus system}

First, consider a two-bus system having one voltage source and one load bus (modeled as an impedance load) as shown in Fig. 1 . Let $E=1.1 \mathrm{pu}, \theta$ change from $10^{\circ}$ to $40^{\circ}$, $Z_{T}=0.03 \mathrm{pu}, \beta=80^{\circ}$, and $\bar{S}=1200+\mathrm{j} 400$ (MVA) at $t=0 \mathrm{~s}$. Every 5 s, increase the load $\bar{S}$ by $100+\mathrm{j} 40$ (MVA). Fig. 4 gives the simulation results on the voltage, power factor and the $P, Q$ and $S$ of the load. From Fig. 4(c), $P, Q$ and $S$ reach their maxima (i.e. the transfer limits) at $t=911 \mathrm{~s}, 931 \mathrm{~s}$ and $1066 \mathrm{~s}$, respectively.

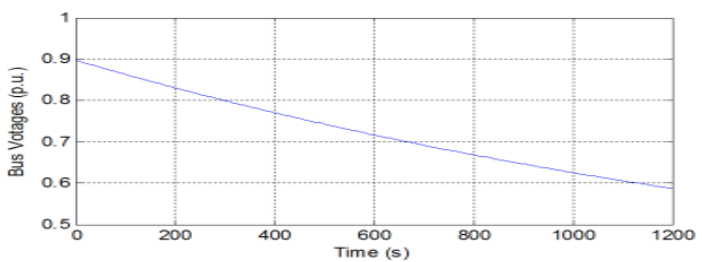

(a) Load bus voltage

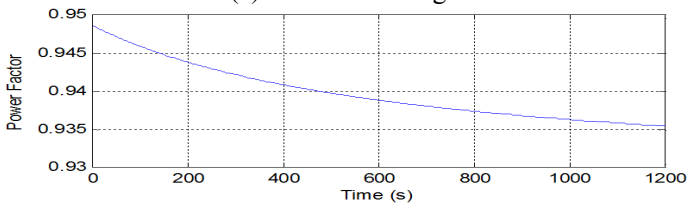

(b) Power factor

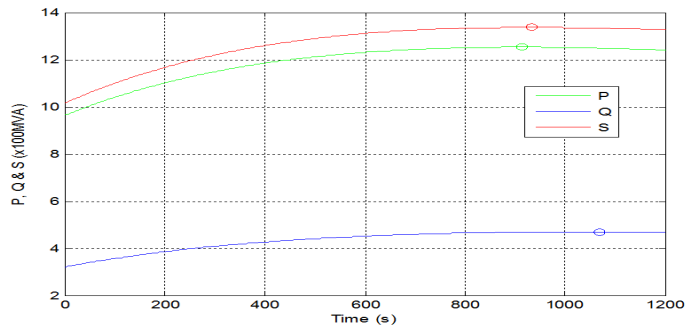

(c) Apparent, real and reactive powers of the load

Fig. 4. Simulation results on a single-generator system

Then, the two approaches are respectively performed every $0.2 \mathrm{~s}$ over the latest $10 \mathrm{~s}$ time window of voltage and current data on the load bus. Calculation results are shown in Fig. 5 Fig. 9, where the results labeled with "real", "old" and "new" are respectively actual values and the results from the old and new approaches. Fig. 5 compares the estimates on $E$ and $V$ and their angles with the actual values. Fig. 6 gives the actual $Z_{T}$, the $Z_{T}$ from the old approaches, the $Z_{T}, Z_{T}^{S}, Z_{T}^{P}$ and $Z_{T}^{Q}$ from the new approach, which are all in the range of 
$0.02-0.035 \mathrm{pu}$ and cross the curve of $Z_{L}$ during $t=900 \sim 1100 \mathrm{~s}$, indicating that $S_{\max }, P_{\max }$ and $Q_{\max }$ are met during that period. Fig. 6(b) zooms in on the impedance magnitudes of Fig. 6(a).

From those figures, the new approach can accurately estimate all Thevenin parameters including $E, \theta, Z_{T}$ and $\beta$. However, the results from the old approach have obvious errors, which are caused by its assumption on constant $\varphi$ and $\theta$ in the 10 s time window. From Fig. 6 Fig. 9, the new approach tells that $P, Q$ and $S$ reach their limits $P_{\max }, Q_{\max }$ and $S_{\max }$ at $t=911 \mathrm{~s}, 931 \mathrm{~s}$ and $1066 \mathrm{~s}$, respectively. That exactly matches the observation from Fig. 4(c). However, the old approach tells that $Z_{L}$ meets $Z_{T}$ at about $t=1000 \mathrm{~s}$, different from any of those three times.
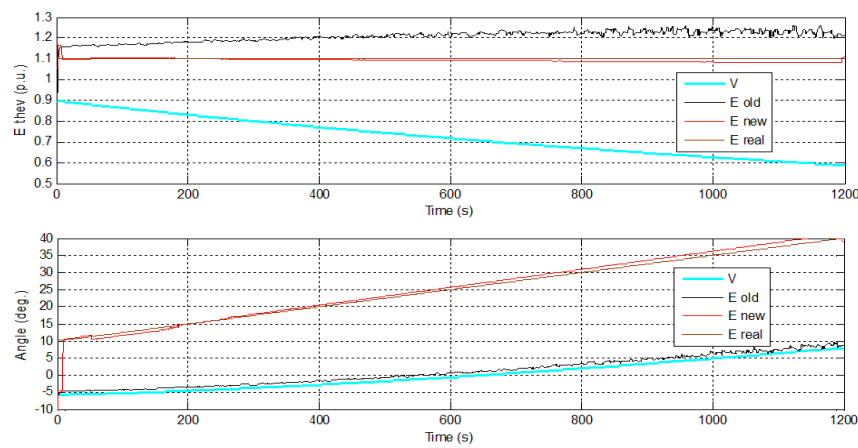

Fig. 5. Estimates on $V$ and $E$ and their angles

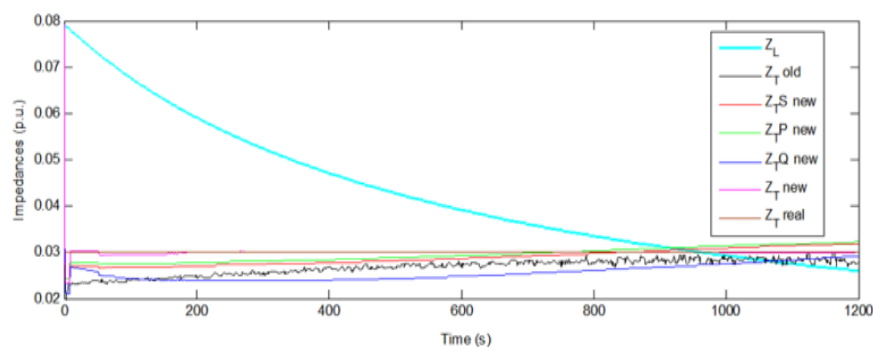

(a) Impedance magnitudes

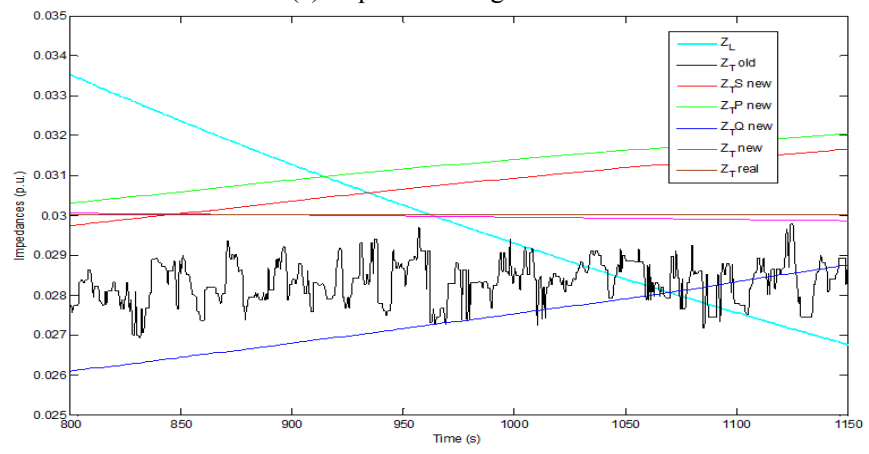

(b) Zoom in on (a)

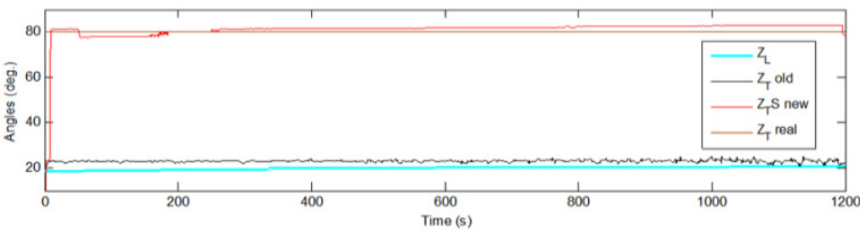

(c) Impedance angles
Fig. 6. Impedance indices

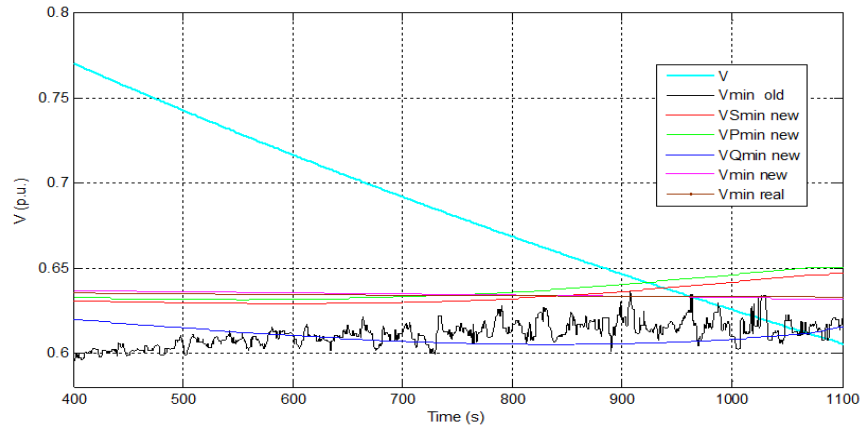

Fig. 7. Voltage indices

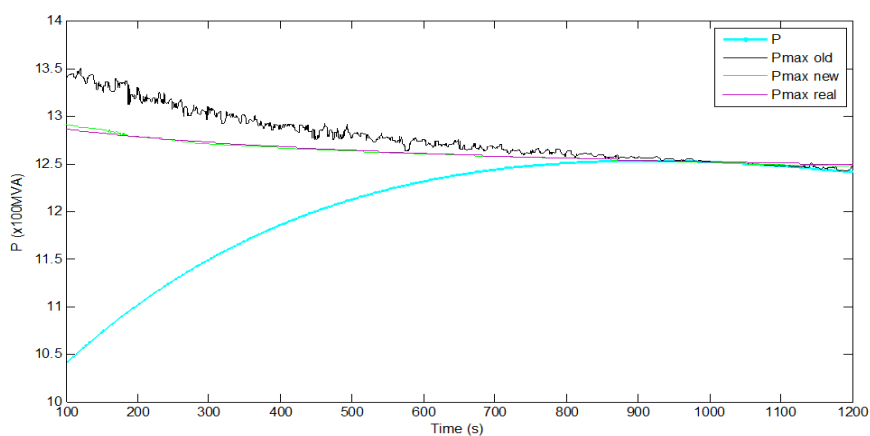

(a) Real power

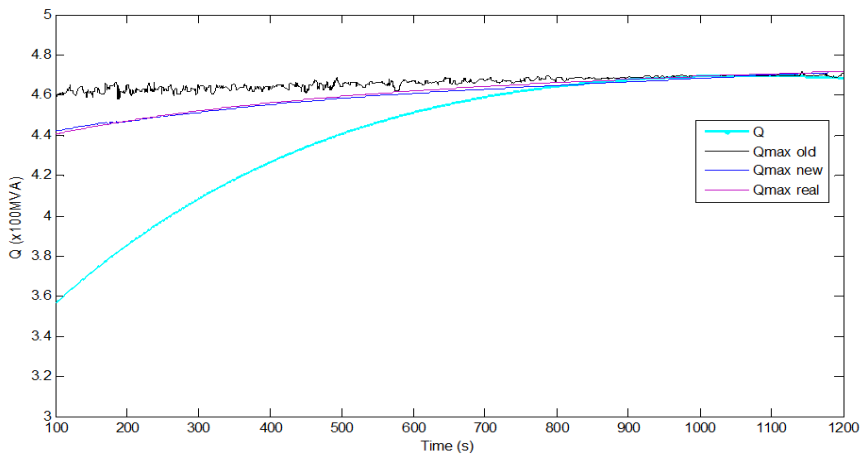

(b) Reactive power

Fig. 8. Power transfer limits

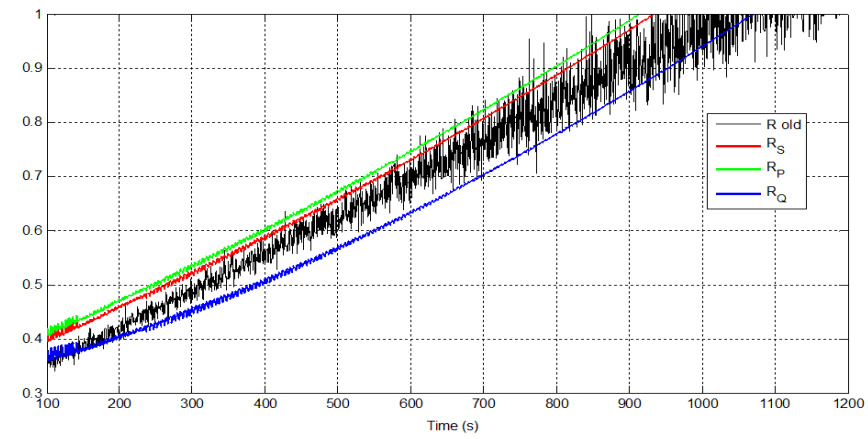

Fig. 9. Risk indices

\section{B. IEEE 39-bus system}

For the IEEE 39-bus system, a load area is defined as indicated by Fig. 10. The load area has three boundary buses, 
i.e. buses 4, 8 and 14, where PMUs are placed. Simulate the following contingency to create a voltage collapse scenario: starting from $\mathrm{t}=0 \mathrm{~s}$, keep increasing the total load of the area from $1898 \mathrm{MW}$ by $6.5 \mathrm{MW}$ every 5 seconds to create slow voltage decay in the area until a voltage collapse happens around $t=423 \mathrm{~s}$, as shown in Fig. 11. Tie-line 9-8 is the most critical line since it has the heaviest loading and bus 8 has the lowest voltage magnitude during the simulation. Fig. 12 gives the total $P, Q$ and $S$ of the three tie lines, from which $P$ reaches its maximum value at $t=396 \mathrm{~s}$, earlier than $Q$ and $S$.

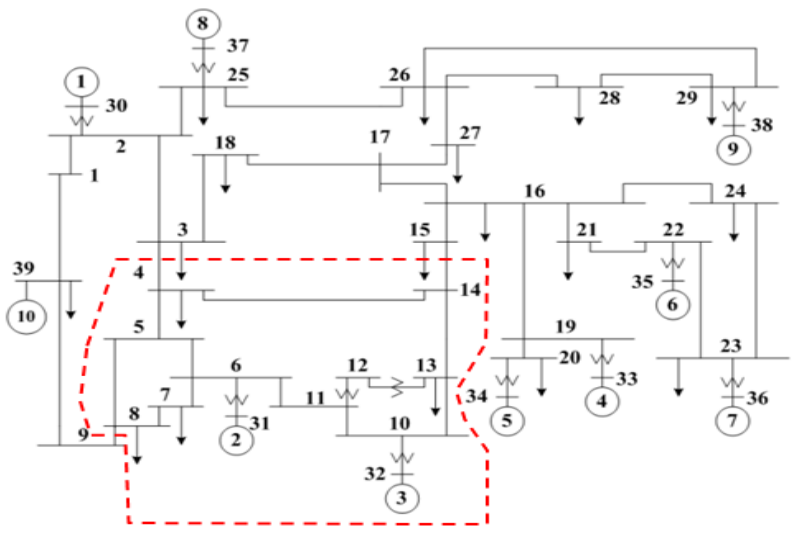

Fig. 10. IEEE 39-bus system

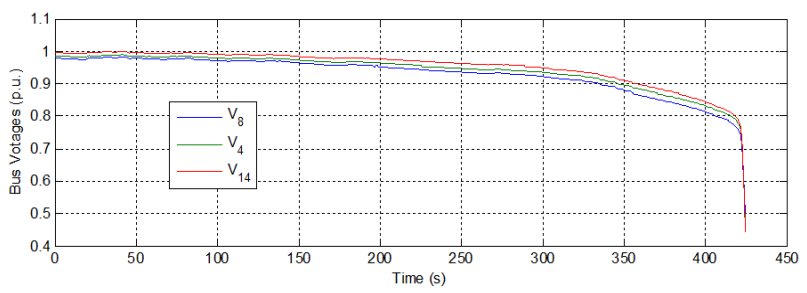

(a) Boundary bus voltage magnitudes

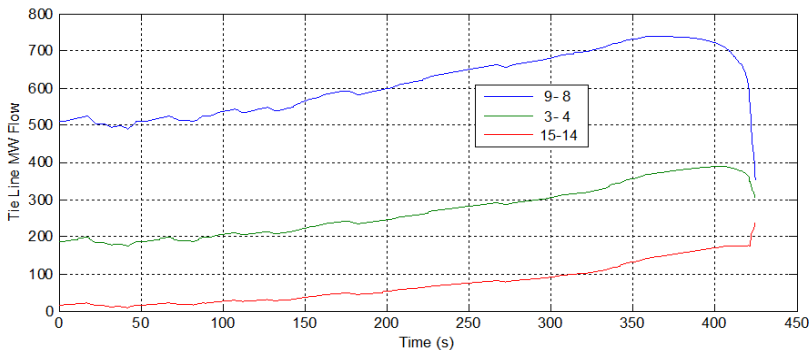

(b) Tie-line MW flows

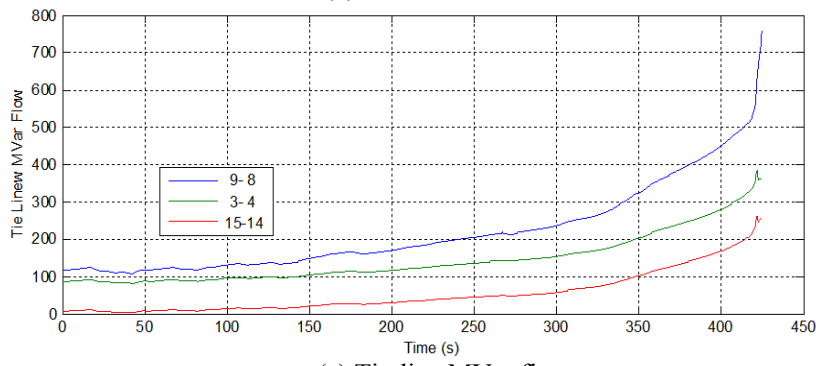

(c) Tie-line MVar flows

Fig. 11. Data on three boundary buses

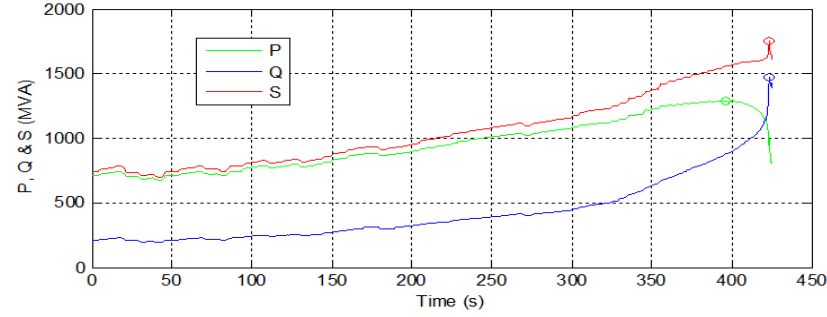

Fig. 12. Total boundary real, reactive and apparent power flows

Calculation results are shown in Fig. 13 Fig. 16. Fig. 13 gives all impedance indices, where $Z_{L}$ meets $Z_{T}^{P}$ at $t=396 \mathrm{~s}$ and $Z_{L}$ meets the $Z_{T}$ of the new approach at $t=423 \mathrm{~s}$, indicating that $P=P_{\max }$ at $396 \mathrm{~s}$ and then voltage collapses at $423 \mathrm{~s}$. That matches the observations from Fig. 12. In Fig. 13, $Z_{L}$ never meets the $Z_{T}$ of the old approach due to the inaccuracy of the old approach on this case. Fig. 14 gives the voltage indices, Fig. 15 gives the limits of the total $P$ and $Q$, and Fig. 16(a) gives the risk indices on the total $P, Q$ and $S$ of the three tie lines. Those figures all confirm that $P=P_{\max }$ at $396 \mathrm{~s}$ and then voltage collapses at 423s. Fig. 14(b) gives the risk indices of three tie lines on the total $P$, from which tie line 9-8 has the highest risk. That matches the observations from Fig. 11.

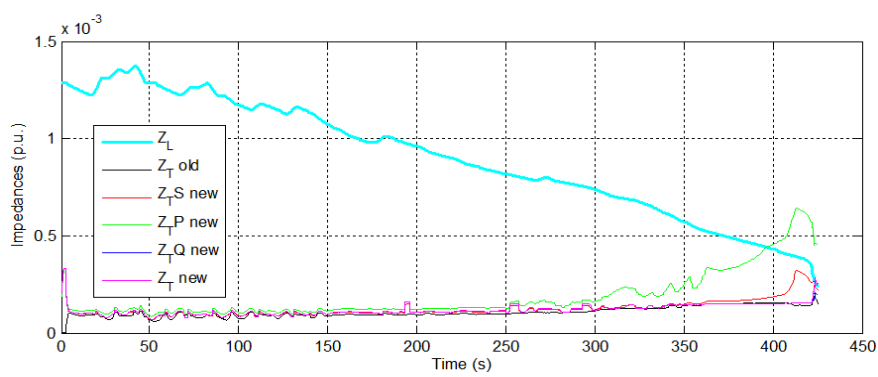

(a) Impedance magnitudes

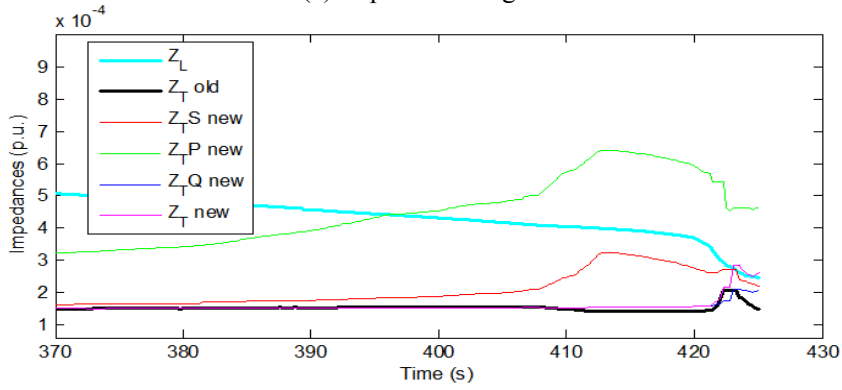

(b) Zoom in on (a)

Fig. 13. Impedance indices

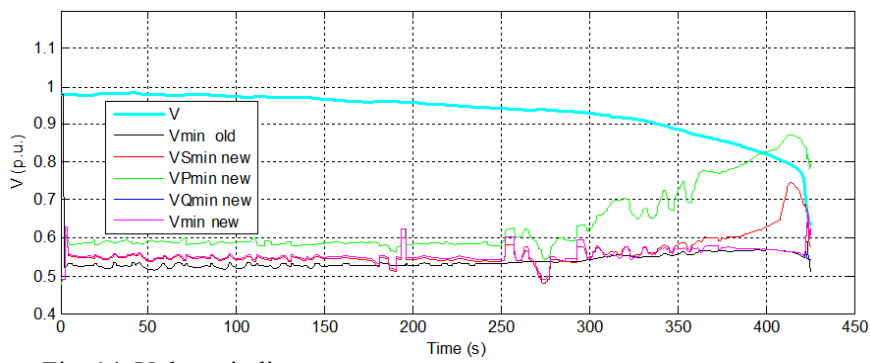

Fig. 14. Voltage indices 


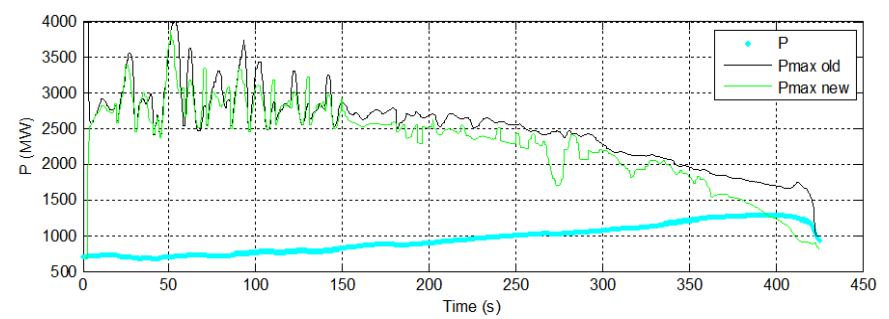

(a) Real power

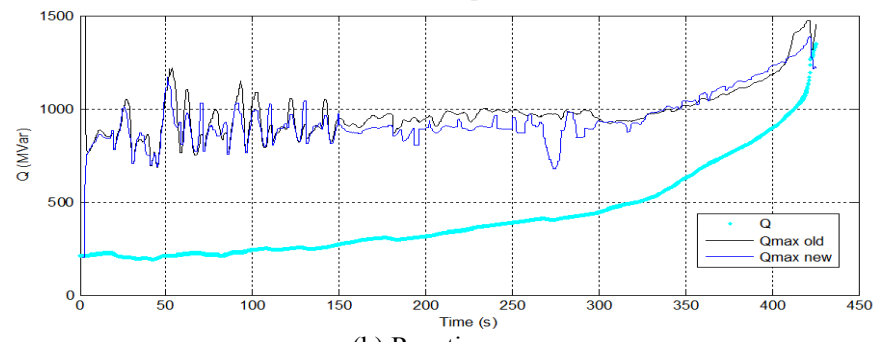

(b) Reactive power

Fig. 15. Power transfer limits

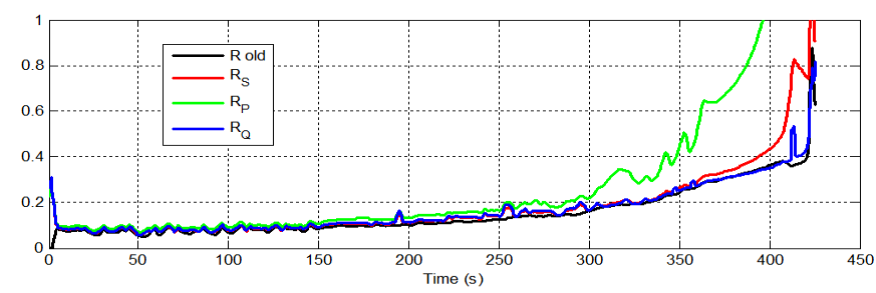

(a) Indices of the total interface

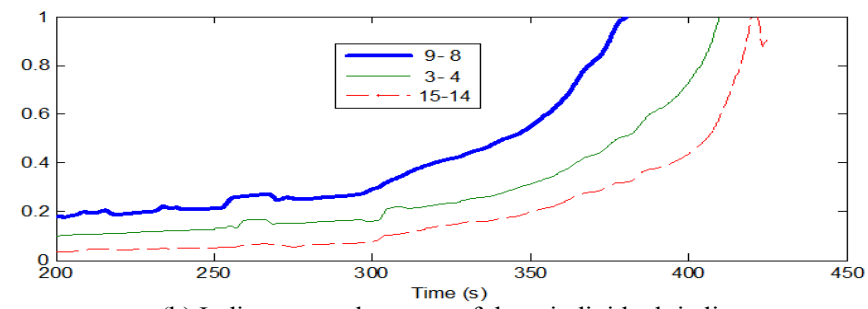

(b) Indices on real powers of three individual tie lines

Fig. 16. Risk indices

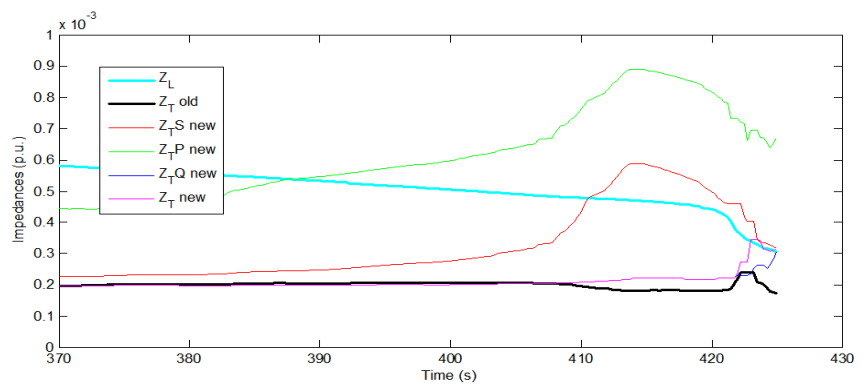

Fig. 17. Impedance indices from PMUs only at boundary buses 4 and 8

For the case that boundary buses and tie lines are only partially monitored by PMUs, the new approach is also tested. Fig. 17 gives the impedance indices calculated using only measurement data on buses 4 and 8 . Note that bus 14 is the least critical one from Fig. 16(b). Fig. 17 tells that the total $P$ of tie lines 9-8 and 3-4 meets the limit at $t=388 \mathrm{~s}$ (where $Z_{L}=$ $Z_{T}^{P}$ ), which is slightly earlier than $396 \mathrm{~s}$ from the monitoring on three tie lines. Fig. 17 also shows that $Z_{L}$ meets the $Z_{T}$ from the new approach around $t=423 \mathrm{~s}$, indicating the voltage collapse at the same time. The above test on two tie lines demonstrates that the new approach can still give important voltage stability margin information for the monitored lines.

The case study results indicate that in a real-time operation environment, when a load area is monitored by the new approach, the stability and risk indices demonstrated by the case studies will be good indicators to provide important voltage stability information in real time including voltage stability margin, the limiting factor $(P$ or $Q)$, and the most critical tie line. That information is valuable to choose effective remedial actions against voltage instability

\section{CONCLUSION}

A new approach is proposed to monitor voltage stability for a load area using PMUs on boundary buses and is tested by case studies. The new approach can provide when the power transfer through monitored tie lines meets real, reactive or apparent power limits. It is more accurate than a traditional Thevenin equivalent-based approach when external phase angles are not directly measurable or the power factors of tie-line flows are not constant.

\section{REFERENCES}

[1] K. Vu, M. Begovic, D. Novosel, and M. Saha, "Use of local measurements to estimate voltage-stability margin," IEEE Trans. Power Systems, vol. 14, no. 3, pp. 1029-1035, Aug. 1999.

[2] B. Milosevic and M. Begovic, "Voltage-stability protection and control using a wide-area network of phasor measurements," IEEE Trans. Power Systems, vol. 18, pp. 121-127, 2003.

[3] Smon, et al, "Local Voltage-Stability Index Using Tellegen's Theorem," IEEE Trans. Power Systems, Vol. 21, No. 3, Aug. 2006.

[4] P. Zhang, L. Min, et al, "Measurement based voltage stability monitoring and control", US Patent \# 8,126,667, Feb 2012.

[5] K. Sun, P. Zhang, L. Min, "Measurement-based Voltage Stability Monitoring and Control for Load Centers", EPRI Technical Report No. 1017798, 2009.

[6] M. Parniani, et al, "Voltage Stability Analysis of a Multiple-Infeed Load Center Using Phasor Measurement Data," IEEE PES Power Systems Conference and Exposition, Nov 2006

[7] S. Corsi and G. Taranto, "A real-time voltage instability identification algorithm based on local phasor measurements," IEEE Trans. Power Systems. vol. 23, no. 3, pp. 1271-1279, Aug. 2008.

[8] M. Glavic and T. Van Cutsem, "Wide-Area Detection of Voltage Instability from Synchronized Phasor Measurements. Part I: Principle," IEEE Trans. Power Systems, vol. 24, pp. 1408 - 1416, 2009.

[9] F. Galvan, A. Abur, K. Sun, et al, "Implementation of Synchrophasor Monitoring at Entergy: Tools, Training and Tribulations", IEEE PES General Meeting, 23-26 July 2012, San Diego

[10] F. Hu, K. Sun, N. Bhatt, "An Adaptive Three-bus Power System Equivalent for Estimating Voltage Stability Margin from Synchronized Phasor Measurements", IEEE PES General Meeting, July 27-31, 2014, Washington, DC

[11] R. Diao, K. Sun, V. Vittal, et al, "Decision Tree-Based Online Voltage Security Assessment Using PMU Measurements", IEEE Trans. Power Systems, vol. 24, pp.832-839, May 2009. 